\title{
Trapping methods for probing functional intermediates in nitric oxide synthases and related enzymes
}

DOI:

$10.2741 / 4678$

\section{Document Version}

Accepted author manuscript

Link to publication record in Manchester Research Explorer

\section{Citation for published version (APA):}

Hedison, T., Hay, S., \& Scrutton, N. (2018). Trapping methods for probing functional intermediates in nitric oxide synthases and related enzymes. Frontiers in Bioscience, 23(10), 1874-1888. https://doi.org/10.2741/4678

\section{Published in:}

Frontiers in Bioscience

\section{Citing this paper}

Please note that where the full-text provided on Manchester Research Explorer is the Author Accepted Manuscript or Proof version this may differ from the final Published version. If citing, it is advised that you check and use the publisher's definitive version.

\section{General rights}

Copyright and moral rights for the publications made accessible in the Research Explorer are retained by the authors and/or other copyright owners and it is a condition of accessing publications that users recognise and abide by the legal requirements associated with these rights.

\section{Takedown policy}

If you believe that this document breaches copyright please refer to the University of Manchester's Takedown Procedures [http://man.ac.uk/04Y6Bo] or contact uml.scholarlycommunications@manchester.ac.uk providing relevant details, so we can investigate your claim.

\section{OPEN ACCESS}


This is an un-copyedited author manuscript that has been accepted for publication in the Frontiers in Bioscience. Cite this article as it appears in the Journal of Frontiers in Bioscience. Full citation can be found by searching the Frontiers in Bioscience (Search for articles ) following publication and at PubMed

(http://www.ncbi.nlm.nih.gov/entrez/query.fcgi?CMD=search\&DB=pubmed) following indexing. This article may not be duplicated or reproduced, other than for personal use or within the rule of "Fair Use of Copyrighted Materials" (section 107, Title 17, U.S. Code) without permission of the copyright holder, the Frontiers in Bioscience. From the time of acceptance following peer review, the full final copy edited article of this manuscript will be made available at https://www.bioscience.org/. The Frontiers in Bioscience disclaims any responsibility or liability for errors or omissions in this version of the un-copyedited manuscript or in any version derived from it by the National Institutes of Health or other parties. 
Manchester Institute of Biotechnology and School of Chemistry, The University of Manchester, Manchester, United Kingdom.

\title{
TABLE OF CONTENTS.
}

\author{
1. Abstract \\ 2. Introduction \\ 3. Probing internal electron transfer in NOS \\ 3.1. Trapping intermediates during NOS flavin reduction \\ 3.2. Trapping intermediates during the FMN to haem electron transfer step in NOS catalysis \\ 4. Artificial NOS conformational states \\ 5. Exploring the NOS conformational equilibrium with hydrostatic pressure \\ 6. Trapping intermediates at the NOS catalytic core \\ 7. Conclusions \\ 8. Acknowledgements \\ 9. References
}

\section{ABSTRACT}

The three mammalian isoforms of nitric oxide synthase (NOS) produce the signalling molecule nitric oxide (NO) from L-arginine, molecular oxygen, and NADPH. NOS-generated NO is essential for many key biochemical processes and aberrant NO production is linked to the pathophysiology of many diseases. Over the past 40 years, the mechanism and structure of NOS have been studied extensively and there are many quality reviews and perspectives documenting the current hypotheses in the field. In this review, as an alternative perspective, we will describe some novel techniques and methodologies that have been used to trap functionally relevant conformational and kinetic states in the catalytic cycles of NOS and some structurally related diflavin oxidoreductase. The methods described in this perspective have enabled characterisation of the complex NOS and diflavin oxidoreductase family members (including cytochrome P450 reductase) and should find future application in other enzyme systems.

\section{INTRODUCTION}

Much of our understanding of enzyme function is derived from research devoted to enzyme chemical reactions and analysis of static three-dimensional images of individual enzyme molecules. However, due to their dynamic properties and complex reaction mechanisms, multicentre redox enzymes are often challenging to study using these conventional spectroscopic and structural biochemical techniques. To overcome the lack of mechanistic insight from these approaches, more sophisticated methods have been developed. One such method is trapping. Trapping is an umbrella term that can be used to describe any technique where short-lived intermediates, normally found during enzyme turnover, are artificially poised and studied. Trapping is usually achieved by conformational and/or chemical perturbations on the enzyme system of interest, and enables the study of transient states that are usually hidden in complex structural and kinetic data.

Methods of trapping and probing intermediates have been used extensively in the study of numerous different protein systems and have provided detailed insights into enzyme reaction mechanisms. A family of enzymes that have been studied using a variety of different trapping methods is the diflavin oxidoreductases. Diflavin oxidoreductases are structurally homologous multidomain electron transferases that contain tightly bound FAD and FMN cofactors (1-3). Members of the diflavin oxidoreductases include cytochrome P450 reductase (CPR) (4-6), methionine synthase reductase (MSR) (7, 8), sulphite reductase (SiR) (9), cytochrome P450 BM3 from Bacillus megaterium (P450 BM3) (10), novel reductase 1 (NR1) (11), and the three isoforms of mammalian nitric oxide synthase (NOS) (12-14). Due to their potent physiological roles (e.g. blood pressure regulation $(15,16)$ and neurogenesis $(17-19))$, the isoforms of mammalian NOS are one of the more studied diflavin oxidoreductases. In this review, we will show how trapping experiments have helped to elucidate and provide invaluable insight into the mechanism of NOSs and the structurally related diflavin oxidoreductases.

Since its discovery as the mammalian enzyme that produces nitric oxide (NO), NOS has been extensively studied (20). At a basic level, NOS functions by shuttling electrons from NADPH, through FAD and FMN cofactors, to a catalytic haem domain where L-arginine and dioxygen are converted into L-citrulline and NO (Figure 1) (12-14, 20). However, like many other multicentre redox enzymes, NOS electron transfer chemistry is complex and thought to be gated by protein domain dynamics $(12,21)$. To study the NOS electron transfer chemistry and conformational changes, several real-time (4, 22-24), novel structural biology (e.g. EPR (25-27), mass spectrometry (28), cryo-EM (29-31), and fluorescence (32-40)), and trapping techniques have been used. Together, this combinatory approach to study the mechanism of an enzyme has shown how a finely tuned temporal and spatial domain 'dance', coordinated by enzyme redox chemistry, NADPH and calmodulin $(\mathrm{CaM})$ binding, regulates the flow of electrons through the NOS family of enzymes.

The three isoforms of NOS are each located on different chromosomes, expressed in different tissues and have different primary amino acid sequences (20). The constitutively expressed NOS isoenzymes (cNOS), neuronal NOS (nNOS) 
and endothelial (eNOS), like their names suggest, are expressed in neuronal and endothelial tissues, respectively, whilst the inducible NOS (iNOS) enzyme is not expressed under normal physiological conditions, but is produced in any tissue type in immune responses. Despite these differences, the three NOS isoforms have very similar catalytic mechanisms $(12,41,42)$ and structures (29-31). NOS is a homodimer, with each monomer containing a reductase and an oxygenase portion. The NOS reductase is similar to other members of the diflavin oxidoreductase enzyme family and contains an FAD-binding domain and an FMN-binding domain, separated by a connecting domain. The N-terminal NOS oxygenase domain is the catalytic core of the enzyme, as well as being the site of enzyme dimerisation, and contains a Cys-ligated $b$-haem and a tetrahydrobiopterin $\left(\mathrm{H}_{4} \mathrm{~B}\right)$ cofactor. Lying between the NOS reductase and oxygenase portions is a CaM binding site.

The current hypothesis for the mechanism of NOS is reviewed in some of our recently published perspectives ( 2 , 12,40 ), and a schematic representation is presented in Figure 2. In the absence of the coenzyme and/or CaM, NOS samples multiple different conformations (in the resting state), with little to no functional relevance. Upon binding of the reduced coenzyme, NADPH, the FAD and FMN domains of NOS lock together. Locking of the FAD and FMN domains in the NADPH-bound state is crucial for interflavin electron transfer and occurs in the cNOS isoenzymes due to interactions between the NADPH coenzyme and several important amino acids regions of the protein, termed the auto-inhibitory insert (AI) (43-45) and the NOS C-terminal tail (CT) (46-49). $\mathrm{Ca}^{2+}$-bound CaM interactions with NOS free the locked input state $(47,48,50-52)$, enabling the FMN domain to shuttle between the FAD and the haem domain and drives cross-monomer electron transfer $(53,54)$ from the NOS reductase to the NOS oxygenase.

Among many other experimental techniques, trapping methods have been invaluable to the study of the NOS family of enzymes and the structurally related diflavin oxidoreductases, and have helped to shape our current understanding of NOS catalysis. The examples of trapping described in this review include methods of deconvoluting complex spectroscopic data for simpler analysis of both chemical and conformational chemistry, as well as illustrating how conformational perturbations can influence and, thus, help determine the mechanism of NOS. Many of the techniques detailed herein are innovative and could find future applications in the study of other multicentre redox enzymes and dynamic protein systems.

\section{PROBING INTERNAL ELECTRON TRANSFER IN NOS}

As NOS contains multiple cofactors with overlapping redox-dependent spectral features, the study of NOS reaction chemistry by transient kinetic methods is challenging. To overcome these complexities, a number of different biochemical approaches have been utilised. One such method of probing NOS redox chemistry has been to deconstruct NOS into individual domains or portions allowing discrete kinetic steps to be probed in isolation. Multiple comprehensive kinetic studies on NOS have been performed using this 'stripped-down' approach, including studies on the isolated NOS reductase portion (using stopped-flow methods) to analyse flavin reduction (23), and studies on the NOS oxygenase-FMN (oxyFMN) domain construct (using laser flash photolysis methods) to monitor electron transfer from the NOS FMN to the haem oxygenase domain $(55,56)$. These studies have been instrumental in understanding the mechanism of NOS. Nevertheless, due to the role of neighbouring domains influencing protein dynamics (25) and, thus, reaction chemistry, the kinetics observed in these deconstructed portions may not accurately represent the reaction chemistry of the full-length enzyme.

Various trapping approaches have also been used to probe redox chemistry in the full-length NOS enzymes, as well as in the truncated NOS constructs. Trapping can be advantageous in the study of enzyme catalysed electron transfer reactions, and is usually carried out by placing carefully designed 'thermodynamic blocks' along the enzyme reaction coordinate (either by mutagenic techniques, adding/replacing small molecules or using temperature perturbation). Through the use of trapping methods, complex spectroscopic data interpretation is often simplified.

\subsection{Trapping intermediates during NOS flavin reduction}

Full length NOS flavin reduction was recently studied using trapping approaches (22). NOS flavin reduction is typically monitored in a stopped-flow instrument by mixing oxidised enzyme with NADPH, and following flavin absorbance bands at $485 \mathrm{~nm}$ (a haem isosbestic point) (22). However, as stopped-flow traces observed for NOS flavin reduction are complex and multiphasic (Figure 3B), it is difficult to assign observed rate constants to discrete chemical steps. Therefore, for deeper insight into NOS flavin redox kinetics, we recently utilised a novel approach that involved the use of a flavin analogue. By partially unfolding and refolding nNOS in these studies, we were able to swap the NOS FMN cofactor for a 5deaza flavin mononucleotide (5-dFMN) molecule, and produce a new form of NOS (5-dFMN nNOS). Unlike nNOS, 5dFMN nNOS was unable to catalyse interflavin electron transfer (FAD $\rightarrow$ FMN electron transfer) but maintained similar secondary structure to the wild-type enzyme. This follows because of the properties of 5-dFMN. 5-dFMN is a flavin biomimetic that, while being isostructural to normal FMN, is an obligate 2-electron redox cofactor incapable of stabilising 1electron reduced forms (flavin semiquinone states) essential for NOS interflavin electron transfer (Figure 2) (57).

Stopped-flow transients observed for 5-dFMN nNOS flavin reduction (with and without CaM) are comparable to those observed for the wild-type enzyme (Figure 3B). Specifically, observed rate constants and amplitude changes reporting on the first three kinetic phases $\left(k_{\mathrm{obs} 1}, k_{\mathrm{obs} 2}, k_{\mathrm{obs} 3}\right)$ associated with flavin reduction are alike in nNOS and 5-dFMN nNOS. However, unlike nNOS, the fourth slower kinetic phase $\left(k_{\mathrm{obs} 4}\right)$ is absent in studies with 5-dFMN NOS. As 5-dFMN is unable to transfer electrons from FAD to FMN, this suggests that this slower fourth phase is predominantly associated with interflavin electron transfer. Altogether, in these studies, through the use of 5-dFMN, we were able to simplify complex 
transients observed for NOS flavin reduction, and to correlate individual kinetic phases with specific steps in NOS chemical catalysis (Figure 3).

\subsection{Trapping intermediates during the FMN to haem electron transfer step in NOS catalysis}

In the early 1990s, there was much debate and discussion over the control and mechanism of NOS haem reduction. Work conducted during this period lead to a number of seminal publications emphasising the role of key elements that drive electron transfer from the NOS reductase to the NOS oxygenase, and laid the foundations for a series of trapping experiments to investigate inter- or intra-subunit electron transfer in the NOS family of enzymes. For electron transfer from the NOS FMN hydroquinone to the NOS oxygenase, the enzyme requires the (i) substrate L-arginine and the cofactor $\mathrm{H}_{4} \mathrm{~B}$, (ii) the presence of $\mathrm{CaM}$ (52) and (iii) the enzyme to be in a dimeric form. L-arginine and $\mathrm{H}_{4} \mathrm{~B}$ binding alters the environment of the haem porphyrin and increases the redox potential of the haem iron (58), while CaM binding shifts the conformational equilibrium of NOS to the 'output' state and modifies the midpoint potentials of the flavin cofactors $(22,25$, 39, 59). The latter mentioned feature, NOS dimerisation, is essential for haem reduction as L-arginine does not bind to the monomeric form of NOS and electron transfer from NOS FMN to haem is thought to occur in a cross-monomer (intermolecular) fashion $(53,54,60)$.

As cross-monomer electron transfer is often observed in multimeric electron transferases and can provide additional control over enzyme-catalysed reaction chemistry (e.g. through oligomerisation steps) (61), it is not difficult to envision why certain electron transfer steps in NOS catalysis could occur from one monomer to the other. Several trapping experiments have previously been designed and conducted by both the Stuehr $(54,60)$ and Sagami $(53)$ groups to test the implications of NOS domain swapping. In these investigations, the authors generated a library of NOS heterodimeric constructs, containing one full-length NOS monomer and one NOS oxygenase domain. By using a mixture of wild-type NOS and an L-arginine binding knock-out variant, the authors could determine the pathway of electron transfer from NOS reductase to oxygenase by using steady state NO production assays (Figure 4). This approach of using mutagenesis to 'block' electron transfer in the NOS enzyme is an example of how trapping measurements have been useful in probing electron transfer pathways and has found wider application in the study of other diflavin oxidoreductases, such as P450 BM3 (62).

To accurately measure the kinetics of electron transfer from FMN to haem in NOS, laser flash photolysis methods have been developed $(55,56,63-65)$. The laser flash photolysis method works by rapid injection of electrons into redox centres and can remove the complexities of slow mixing and multiple overlapping transients observed using stopped-flow methods. Carbon monoxide trapping methods have been used to study FMN to haem electron transfer in NOS. By flashing $\mathrm{CO}$ off partially reduced [Fe(II)-CO][FMNH'] form of NOS, electron transfer in the non-physiological direction from haem to FMN can be monitored. As the NOS $\mathrm{Fe}(\mathrm{II}) / \mathrm{Fe}(\mathrm{III})$ and $\mathrm{FMNH}^{*} / \mathrm{FMNH}_{2}$ couples are near isopotential, electron transfer between the $\mathrm{Fe}(\mathrm{II})$ and $\mathrm{FMNH}^{\circ}$ in NOS is reversible. Therefore, observed rate constants using this method are the sum of both the forward and reverse reaction and, thus, rates of electron transfer in the physiological direction are half of those observed. In short, through the use of these CO-trapping methods, the importance of CaM binding, the AI loop (66) and domain dynamics have been shown in NOS catalysis.

\section{ARTIFICIAL NOS CONFORMATIONAL STATES}

There is evidence from crystallographic data that protein dynamics are required for diflavin oxidoreductase function (6). In the case of NOS family of enzymes, structures of the wild-type reductase construct in complex with NADP ${ }^{+}$ show the FAD and FMN cofactors of the enzyme are in close proximity, and that large-scale motions (50-100 $\AA$ ) of the FMN-binding domain are required for electron transfer from the NOS reductase to the NOS oxygenase (46). Here, to illustrate the importance of NOS conformational change, we focus on a number of specific experimental approaches, where methods to restrict the conformational equilibrium have proven to be useful in the study of NOS (and other diflavin oxidoreductase) function.

Cytochrome P450 reductase (CPR), a member of the diflavin oxidoreductases, which activates the superfamily of drug detoxifying P450 proteins, has been studied using a number of conformational trapping techniques. CPR is structurally homologous to the NOS reductase and, like NOS, is proposed to cycle between 'closed' and 'open' states, with short and long distances between the flavin cofactors, respectively $(4,67,68)$. An artificially closed variant of CPR has been constructed (69). This closed CPR is Cys-Cys disulphide cross-linked and is homologous in structure to the wild-type enzyme (6), with a 4-5 $\AA$ distance between the isoalloxazine moieties of the flavin cofactors (Figure 5). Kinetic studies of this variant have helped show how the 'closed' conformation of the enzyme is functionally relevant for catalysing interflavin electron transfer but cannot support electron transfer from CPR to a partner protein (69).

By removal of key residues in the linker region of CPR ( $\triangle \mathrm{TGEE} \mathrm{CPR),} \mathrm{the} \mathrm{structure} \mathrm{of} \mathrm{the} \mathrm{once} \mathrm{elusive} \mathrm{'open'}$ form of CPR has been determined by X-ray crystallography (Figure 5) (70). The distances between the dimethyl benzene rings of FAD and FMN in the structures of open CPR range from 29-60 ̊ (three structures determined for the 'open' state of CPR). Unlike the disulfide locked closed form of CPR, this artificially open variant is unable to catalyse the transfer of electrons from FAD to FMN, but can undergo interprotein electron transfer from the CPR FAD to CYP partner proteins. Since this initial open state CPR study, a variety of open conformers of CPR have also been constructed. These include a yeast-human chimeric CPR protein (71), which has a $84 \AA$ edge-to-edge distance between the two flavin cofactors, and a the 
structure of the $\triangle$ TGEE variant of CPR in complex with haem oxygenase (HO) (72), which shows a $30 \AA$ distance between the FAD and FMN cofactors and a $6 \AA$ distance between the FMN of CPR and the haem of haem oxygenase. Taken together, these data suggest the 'closed' form of CPR is required for inter-flavin electron transfer while the 'open' form of the enzyme is needed for CPR-partner protein electron transfer.

Recently, a novel trapping method has been developed to study the role of large-scale domain motions in NOS catalysis (73). Through the use of varying length bis-maleimides, the conformational freedom of the NOS reductase domains was restricted to varying degrees. By using shorter linkers, NOS was forced into a more closed state with short distance between the flavin cofactors, while through the use of long linkers the NOS enzyme was constrained to more open states. Flavin reduction rates were seen to increase in the more closed forms of the enzyme when comparing to wild-type protein (flavin reduction limited by protein dynamics), while both single turnover and steady state cytochrome $c$ reduction rates were significantly impaired in this closed state. As expected, in this experiment, as the tether length was increased, the rates of interflavin electron transfer and interprotein electron transfer were decreased and increased, respectively. As no tethered enzyme could outperform wild-type NOS steady-state cytochrome $c$ reduction rates in this investigation, it was suggested that motions of the FMN domain are essential for NOS catalysis. This technique and the two aforementioned studies on CPR have helped build up a now largely consistent model of NOS catalysis, showing the importance of both 'closed' and 'open' conformations and the need for conformational freedom to shuttle electrons from NADPH to the NOS catalytic haem domain.

\section{EXPLORING THE NOS CONFORMATIONAL EQUILIBRIUM WITH HYDROSTATIC PRESSURE}

Complementary to conformational trapping approaches is the use of hydrostatic pressure to reversibly control the conformations of multidomain proteins such as the diflavin reductases $(25,68)$. Pressure acts on underlying equilibria, perturbing the system towards more compact states at higher pressures. As a result, it is possible to shift the position of $e . g$. an open/closed (or bound/free) conformational equilibrium by raising the pressure. A range of experiment apparatuses are available that allow measurement of spectroscopic features (e.g. UV-Vis, FTIR or NMR spectra) of proteins under a range of moderate hydrostatic pressures up to $2-3,000$ atmospheres $(74,75)$. Above these pressures, many proteins denature due to a breakdown of the hydrophobic effect through water ingress into the protein core (76). Transient experiments can also be performed with high pressure stopped-flow, flash photolysis and pressure jump instruments.

We have performed stopped-flow measurements of flavin reduction in CPR over a range of pressures between 12,000 atmospheres (68). Two kinetic phases are observed and both are moderately pressure dependent. It can be difficult to predict which conformational state(s) are likely to be more compact and thus favoured at high pressure, but in the case of CPR, surface area calculations suggest closed conformation(s) are likely to be more compact (Figure 5). As flavin reduction occurs more quickly at high pressure, these data are consistent with a model where inter-flavin electron transfer is conformationally gated and occurs more rapidly under conditions when CPR adopts compact closed conformation(s) with shorter inter-flavin distances. The same behaviour is not observed in nNOS, where flavin reduction steps are largely pressure invariant and/or occur more slowly at higher pressure (25). The rate of steady state NADPH consumption by nNOS also decreases with pressure unless $\mathrm{CaM}$ is removed, at which point the (much slower) rate of consumption significantly increases with pressure. The conformational landscape of NOS is likely to be more complicated (involving more states) than that of CPR, but the nNOS data suggest that pressure induces the adoption of more catalytically competent conformation(s) of CaM-free nNOS, which may be deleterious to FAD reduction and/or interflavin electron transfer when CaM is bound. This could be interpreted such that the conformational landscape of nNOS is relatively optimised for catalysis, so perturbations by high pressure can only push the enzyme into conformations that are less catalytically competent.

\section{TRAPPING INTERMEDIATES AT THE NOS CATALYTIC CORE}

The haem oxygenase domain of NOS is the catalytic core of the enzyme (77-79). Here, through the sequential transfer of two electrons from FMN to haem, L-arginine is oxidised to generate L-citrulline and NO (20). Figure 1B shows the eleven steps required for NOS catalysed NO production (80). To initiate NO production, electrons are transferred from the reduced FMN to the ferric haem, producing the ferrous haem state (step 1). Once generated, ferrous haem binds a molecule of dioxygen, giving the ferric haem-superoxo species (step 2) (81), which is subsequently reduced by the electron transfer from the $\mathrm{H}_{4} \mathrm{~B}$ cofactor (step 3) (82). The latter of these steps produces the haem-peroxo species (83), which is further protonated to form a haem iron-oxo species (step 4). The NOS haem iron-oxo species reacts directly with L-arginine to produce the N $\omega$-hydroxy-L-arginine (NOHA) intermediate and water, as well as regenerating the NOS ferric haem state (step 5) (83). Like in step 1, the ferric haem is reduced to the ferrous state by electron transfer from the reduced FMN (step 6). Following reduction, steps 2 through 4 are repeated again leading to the formation of the formation of the haem iron-oxo species (step 7-9) (80). This species reacts with the NOHA intermediate leading to the production of ferric haem-NO complex and L-citrulline (step 10). Finally, NO is released from the ferric haem-NO complex completing the catalytic cycle of the enzyme (step 11) $(84,85)$.

Many of the intermediates observed at the NOS haem domain have short half-lives and are difficult to observe using transient kinetic and traditional spectroscopic methods. Therefore, to isolate and spectroscopically characterise reactive states, low-temperature approaches (among other approaches, e.g. mutagenesis (86) and hydrostatic pressure (87)) have been used. Examples of these studies include optical characterisation of the NOS ferric haem-superoxo species, at 243 $\mathrm{K}(81)$, determining the redox role of $\mathrm{H}_{4} \mathrm{~B}$, at $243 \mathrm{~K}(88)$, and detection of the haem-peroxo species temperatures, at $77 \mathrm{~K}$ 
(83). Here, we focus on the latter example, in which Davydov et al. have used radiolytic cryoreduction in combination with electron paramagnetic resonance (EPR) to trap and characterise a previously undetected NOS catalytic intermediate (83). This approach of trapping active oxygen intermediates has been developed and used to study haem monooxygenases systems by the Hoffman lab (89). In these investigations, the enzyme ferric haem-superoxo species is reduced with X-ray, gamma or ${ }^{32} \mathrm{P}$ radiation below temperatures of $77 \mathrm{~K}$, trapping the enzyme in the haem-peroxo form. By stepwise increase in temperature, conversion from the haem-peroxo form to the succeeding intermediate can be observed. Through the use of this method, the Hoffman lab has determined previously hidden intermediates in the catalytic cycle of numerous haem oxygenases, including P450 proteins, bacterial NOS and haem oxygenase (89). Studies performed on the mammalian NOS enzyme systems in complex with the substrates L-arginine or NOHA and a non-catalytic $\mathrm{H}_{4} \mathrm{~B}$ analogue (83) showed the first evidence of the NOS haem-peroxo species. It was observed that the NOS haem-peroxo species converts directly to the 'compound 1'-like haem iron-oxo species, and not the haem-hydroperoxo species (as seen with many other haem monooxygenase systems). This result is likely a reflection of the hydrogen-bonding network that provides proton delivery to the oxyhaem and is not direct evidence that this intermediate is not present in the catalytic cycle of NOSs.

\section{CONCLUDING REMARKS}

In this review, we have shown a toolbox of trapping techniques that have been used to study the mechanism of dynamic multicentre enzyme systems. Specifically, we have shown how site-directed mutagenesis can be used to probe electron transfer chemistry and restrict the conformational freedom of the NOS enzyme. Moreover, examples detailed in this review include the use of small molecule 'thermodynamic blocks', which can be used to poise and study reaction chemistry, and the use of pressure and temperature to control NOS conformational and chemical states. Along with real-time methods and novel structural biology techniques, the approaches detailed within here have helped to give a now largely consistent model of NOS catalysis, which illustrated the importance of large-scale protein domain dynamics in catalysis (Figure 2). Nonetheless, for a complete understanding of NOS turnover, there are still a number of important points that need to be addressed. An atomistic structure of the full-length NOS enzyme would provide invaluable insights into the NOS mechanism. Moreover, as much of our understanding of NOS catalysis is derived from in vitro experiments, there is a need to understand how an in-cell setting would influence NOS catalysis. It has recently been shown that the cell environment greatly influences the stability and dynamic properties of individual proteins. As dynamics play a crucial role in NOS catalysis, we propose that an in vivo setting will impact the catalytic cycle of the NOS enzymes. Therefore, for greater insights into the mechanism of NOS, novel in-cell biophysics and biochemical techniques must be developed.

\section{ACKNOWLEDGEMENTS}

The UK Biotechnology and Biological Sciences Research Council (BBSRC) fund the work conducted in the corresponding author's laboratory.

\section{REFERENCES}

1. T. Iyanagi, C. Xia and J. P. Kim: NADPH-cytochrome P450 oxidoreductase: prototypic member of the diflavin reductase family. Archives of Biochemistry and Biophysics, 528(1), $72-89$ (2012)

$2 . \quad$ C. R. Pudney, D. J. Heyes, B. Khara, S. Hay, S. E. J. Rigby and N. S. Scrutton: Kinetic and spectroscopic probes of motions and catalysis in the cytochrome P450 reductase family of enzymes. FEBS Journal, 279(9), 1534-1544 (2012)

3. L. Aigrain, F. Fatemi, O. Frances, E. Lescop and G. Truan: Dynamic control of electron transfers in diflavin reductases. International Journal of Molecular Sciences, 13(11), 15012-15041 (2012)

4. T. M. Hedison, S. Hay and N. S. Scrutton: Real-time analysis of conformational control in electron transfer reactions of human cytochrome P450 reductase with cytochrome c. FEBS Journal, 282(22), 4357-4375 (2015)

$5 . \quad$ D. S. Riddick, X. Ding, C. R. Wolf, T. D. Porter, A. V. Pandey, Q. Zhang, J. Gu, R. D. Finn, S. Ronseaux, L. A. McLaughlin, C. J. Henderson, L. Zou and C. E. Fluck: NADPH-cytochrome P450 oxidoreductase: Roles in physiology, pharmacology, and toxicology. Drug Metabolism and Disposition: the biological fate of chemicals, 41(1), 12-23 (2013)

6. M. Wang, D. L. Roberts, R. Paschke, T. M. Shea, B. S. S. Masters and J. J. P. Kim: Three-dimensional structure of NADPH-cytochrome P450 reductase: prototype for FMN- and FAD-containing enzymes. Proceedings of the National Academy of Sciences, USA, 94(16), 8411-8416 (1997)

7. D. Leclerc, A. Wilson, R. Dumas, C. Gafuik, D. Song, D. Watkins, H. H. Q. Heng, J. M. Rommens, S. W. Scherer, D. S. Rosenblatt and R. A. Gravel: Cloning and mapping of a cDNA for methionine synthase reductase, a flavoprotein defective in patients with homocystinuria. Proceedings of the National Academy of Sciences of the United States of America, 95(6), 3059-3064 (1998)

8. S. E. J. Rigby, X. Lou, H. S. Toogood, K. R. Wolthers and N. S. Scrutton: ELDOR spectroscopy reveals that energy landscapes in human methionine synthase reductase are extensively remodelled following ligand and partner protein binding. Chembiochem, 12(6), 863-867 (2011)

9. J. Ostrowski, M. J. Barber, D. C. Rueger, B. E. Miller, L. M. Siegel and N. M. Kredich: Characterization of the flavoprotein moieties of nadph-sulfite reductase from salmonella-typhimurium and escherichia-coli - physicochemical and catalytic properties, amino-acid sequence deduced from DNA-sequence of cysJ, and comparison with NADPH-cytochromeP-450 reductase. Journal of Biological Chemistry, 264(27), 15796-15808 (1989)

10. A. W. Munro, D. G. Leys, K. J. McLean, K. R. Marshall, T. W. B. Ost, S. Daff, C. S. Miles, S. K. Chapman, D. A. Lysek, C. C. Moser, C. C. Page and P. L. Dutton: P450BM3: the very model of a modern flavocytochrome. Trends in Biochemical Sciences, 27(5), 250-257 (2002) 
11. M. J. I. Paine, A. P. Garner, D. Powell, J. Sibbald, M. Sales, N. Pratt, T. Smith, D. G. Tew and C. R. Wolf: Cloning and characterization of a novel human dual flavin reductase. Journal of Biological Chemistry, 275(2), 1471-1478 (2000)

12. N. G. H. Leferink, S. Hay, S. E. J. Rigby and N. S. Scrutton: Towards the free energy landscape for catalysis in mammalian nitric oxide synthases. FEBS Journal, 282(16), 3016-3029 (2015)

13. D. J. Stuehr: Mammalian nitric oxide synthases. Biochimica Et Biophysica Acta-Bioenergetics, 1411(2-3), $217-$ 230 (1999)

14. D. J. Stuehr: Enzymes of the L-arginine to nitric oxide pathway. Journal of Nutrition, 134(10), 2748S-2751S (2004)

15. R. M. Rapoport, M. B. Draznin and F. Murad: Endothelium-dependent relaxation in rat aorta may be mediated through cyclic gmp-dependent protein-phosphorylation. Nature, 306(5939), 174-176 (1983)

16. N. Kim, K. M. Azadzoi, I. Goldstein and I. S. Detejada: A nitric-oxide like factor mediates nonadrenergicnoncholinergic neurogenic relaxation of penile corpus cavernosum smooth-muscle. Journal of Clinical Investigation, 88(1), $112-118$ (1991)

17. T. J. Odell, R. D. Hawkins, E. R. Kandel and O. Arancio: Tests of the roles of 2 diffusible substances in long-term potentiation - evidence for nitric-oxide as a possible early retrograde messenger. Proceedings of the National Academy of Sciences of the United States of America, 88(24), 11285-11289 (1991)

18. E. M. Schuman and D. V. Madison: A requirement for the intercellular messenger nitric-oxide in long-term potentiation. Science, 254(5037), 1503-1506 (1991)

19. L. Zhou and D. Y. Zhu: Neuronal nitric oxide synthase: Structure, subcellular localization, regulation, and clinical implications. Nitric Oxide-Biology and Chemistry, 20(4), 223-230 (2009)

20. W. K. Alderton, C. E. Cooper and R. G. Knowles: Nitric oxide synthases: structure, function and inhibition. Biochemical Journal, 357(Part 3), 593-615 (2001)

21. D. J. Stuehr, J. Tejero and M. M. Haque: Structural and mechanistic aspects of flavoproteins: electron transfer through the nitric oxide synthase flavoprotein domain. FEBS Journal, 276(15), 3959-3974 (2009)

22. T. M. Hedison, N. G. H. Leferink, S. Hay and N. S. Scrutton: Correlating calmodulin landscapes with chemical catalysis in neuronal nitric oxide synthase using time-resolved FRET and a 5-deazaflavin thermodynamic trap. ACS Catalysis, 6(8), 5170-5180 (2016)

23. K. Knight and N. S. Scrutton: Stopped-flow kinetic studies of electron transfer in the reductase domain of neuronal nitric oxide synthase: re-evaluation of the kinetic mechanism reveals new enzyme intermediates and variation with cytochrome P450 reductase. Biochemical Journal, 367(Pt 1), 19-30 (2002)

$24 . \quad$ D. J. Heyes, A. M. Quinn, P. M. Cullis, M. Lee, A. W. Munro and N. S. Scrutton: Internal electron transfer in multi-site redox enzymes is accessed by laser excitation of thiouredopyrene-3,6,8-trisulfonate (TUPS). Chemical Communications, 7(9), 1124-1126 (2009)

25. A. Sobolewska-Stawiarz, N. G. H. Leferink, K. Fisher, D. J. Heyes, S. Hay, S. E. J. Rigby and N. S. Scrutton: Energy landscapes and catalysis in nitric oxide synthase. Journal of Biological Chemistry, 289(17), 11725-11738 (2014)

26. A. V. Astashkin, L. Chen, X. Zhou, H. Li, T. L. Poulos, K. J. Liu, J. G. Guillemette and C. Feng: Pulsed electron paramagnetic resonance study of domain docking in neuronal nitric oxide synthase: the calmodulin and output state perspective. Journal of Physical Chemistry A, 118(34), 6864-6872 (2014)

27. A. V. Astashkin, B. O. Elmore, W. Fan, J. G. Guillemette and C. Feng: Pulsed EPR determination of the distance between heme iron and fmn centers in a human inducible nitric oxide synthase. Journal of the American Chemical Society, 132(34), 12059-12067 (2010)

28. B. C. Smith, E. S. Underbakke, D. W. Kulp, W. R. Schief and M. A. Marletta: Nitric oxide synthase domain interfaces regulate electron transfer and calmodulin activation. Proceedings of the National Academy of Sciences of the United States of America, 110(38), E3577-E3586 (2013)

29. M. G. Campbell, B. C. Smith, C. S. Potter, B. Carragher and M. A. Marletta: Molecular architecture of mammalian nitric oxide synthases. Proceedings of the National Academy of Sciences of the United States of America, 111(35), E3614E3623 (2014)

30. N. Volkmann, P. Martasek, L. J. Roman, X. Xu, C. Page, M. Swift, D. Hanein and B. S. Masters: Holoenzyme structures of endothelial nitric oxide synthase - An allosteric role for calmodulin in pivoting the FMN domain for electron transfer. Journal of Structural Biology, 188(1), 46-54 (2014)

31. A. L. Yokom, Y. Morishima, M. Lau, M. Su, A. Glukhova, Y. Osawa and D. R. Southworth: Architecture of the nitric-oxide synthase holoenzyme reveals large conformational changes and a calmodulin-driven release of the FMN domain. Journal of Biological Chemistry, 289(24), 16855-16865 (2014)

32. A. J. Dunford, S. E. Rigby, S. Hay, A. W. Munro and N. S. Scrutton: Conformational and thermodynamic control of electron transfer in neuronal nitric oxide synthase. Biochemistry, 46(17), 5018-5029 (2007)

33. R. Gachhui, A. Presta, D. F. Bentley, H. M. AbuSoud, R. McArthur, G. Brudvig, D. K. Ghosh and D. J. Stuehr: Characterization of the reductase domain of rat neuronal nitric oxide synthase generated in the methylotrophic yeast Pichia pastoris - Calmodulin response is complete within the reductase domain itself. Journal of Biological Chemistry, 271(34), 20594-20602 (1996)

34. W. Li, W. Fan, B. O. Elmore and C. Feng: Effect of solution viscosity on intraprotein electron transfer between the FMN and heme domains in inducible nitric oxide synthase. FEBS Letters, 585(16), 2622-2626 (2011)

35. H. Li, A. Das, H. Sibhatu, J. Jamal, S. G. Sligar and T. L. Poulos: Exploring the Electron Transfer Properties of Neuronal Nitric-oxide Synthase by Reversal of the FMN Redox Potential. Journal of Biological Chemistry, 283(50), 34762$34772(2008)$ 
36. K. Brunner, A. Tortschanoff, B. Hemmens, P. J. Andrew, B. Mayer and A. J. Kungl: Sensitivity of flavin fluorescence dynamics in neuronal nitric oxide synthase to cofactor-induced conformational changes and dimerization. Biochemistry, 37(50), 17545-17553 (1998)

37. J. C. Salerno, K. Ray, T. Poulos, H. Li and D. K. Ghosh: Calmodulin activates neuronal nitric oxide synthase by enabling transitions between conformational states. FEBS Letters, 587(1), 44-47 (2013)

38. D. K. Ghosh, K. Ray, A. J. Rogers, N. J. Nahm and J. C. Salerno: FMN fluorescence in inducible NOS constructs reveals a series of conformational states involved in the reductase catalytic cycle. FEBS Journal, 279(7), 1306-1317 (2012)

39. Y. He, M. M. Haque, D. J. Stuehr and H. P. Lu: Single-molecule spectroscopy reveals how calmodulin activates NO synthase by controlling its conformational fluctuation dynamics. Proceedings of the National Academy of Sciences of the United States of America, 112(38), 11835-11840 (2015)

40. T. M. Hedison, S. Hay and N. S. Scrutton: A perspective on conformational control of electron transfer in nitric oxide synthases. Nitric Oxide-Biology and Chemistry, 63, 61-67 (2017)

41. R. T. Miller, P. Martasek, T. Omura and B. S. S. Masters: Rapid kinetic studies of electron transfer in the three isoforms of nitric oxide synthase. Biochemical and Biophysical Research Communications, 265(1), 184-188 (1999)

42. L. J. Roman, P. Martasek and B. S. S. Masters: Intrinsic and extrinsic modulation of nitric oxide synthase activity. Chemical Reviews, 102(4), 1179-1190 (2002)

43. J. C. Salerno, D. E. Harris, K. Irizarry, B. Patel, A. J. Morales, S. M. E. Smith, P. Martasek, L. J. Roman, B. S. S. Masters, C. L. Jones, B. A. Weissman, P. Lane, Q. Liu and S. S. Gross: An autoinhibitory control element defines calciumregulated isoforms of nitric oxide synthase. Journal of Biological Chemistry, 272(47), 29769-29777 (1997)

44. S. Daff, I. Sagami and T. Shimizu: The 42-amino acid insert in the FMN domain of neuronal nitric-oxide synthase exerts control over Ca2+/calmodulin-dependent electron transfer. Journal of Biological Chemistry, 274(43), 30589-30595 (1999)

45. H. J. Montgomery, V. Romanov and J. G. Guillemette: Removal of a putative inhibitory element reduces the calcium-dependent calmodulin activation of neuronal nitric-oxide synthase. Journal of Biological Chemistry, 275(7), 5052$5058(2000)$

46. E. D. Garcin, C. M. Bruns, S. J. Lloyd, D. J. Hosfield, M. Tiso, R. Gachhui, D. J. Stuehr, J. A. Tainer and E. D. Getzoff: Structural basis for isozyme-specific regulation of electron transfer in nitric-oxide synthase. Journal of Biological Chemistry, 279(36), 37918-37927 (2004)

47. L. J. Roman, P. Martasek, R. T. Miller, D. E. Harris, M. A. de la Garza, T. M. Shea, J. J. P. Kim and B. S. S. Masters: The $\mathrm{C}$ termini of constitutive nitric-oxide synthases control electron flow through the flavin and heme domains and affect modulation by calmodulin. Journal of Biological Chemistry, 275(38), 29225-29232 (2000)

48. L. J. Roman, R. T. Miller, M. A. de la Garza, J. J. P. Kim and B. S. S. Masters: The C terminus of mouse macrophage inducible nitric-oxide synthase attenuates electron flow through the flavin domain. Journal of Biological Chemistry, 275(29), 21914-21919 (2000)

49. M. Tiso, J. Tejero, K. Panda, K. S. Aulak and D. J. Stuehr: Versatile regulation of neuronal nitric oxide synthase by specific regions of its C-terminal tail. Biochemistry, 46(50), 14418-14428 (2007)

50. D. H. Craig, S. K. Chapman and S. Daff: Calmodulin activates electron transfer through neuronal nitric-oxide synthase reductase domain by releasing an NADPH-dependent conformational lock. Journal of Biological Chemistry, 277(37), 33987-33994 (2002)

51. L. J. Roman and B. S. S. Masters: Electron transfer by neuronal nitric-oxide synthase is regulated by concerted interaction of calmodulin and two intrinsic regulatory elements. Journal of Biological Chemistry, 281(32), 23111-23118 (2006)

52. H. M. Abu-Soud, L. L. Yoho and D. J. Stuehr: Calmodulin controls neuronal nitric-oxide synthase by a dual mechanism. Activation of intra- and interdomain electron transfer. Journal of Biological Chemistry, 269(51), 32047-32050 (1994)

53. I. Sagami, S. Daff and T. Shimizu: Intra-subunit and inter-subunit electron transfer in neuronal nitric-oxide synthase - effect of calmodulin on heterodimer catalysis. Journal of Biological Chemistry, 276(32), 30036-30042 (2001)

54. U. Siddhanta, A. Presta, B. Fan, D. Wolan, D. L. Rousseau and D. J. Stuehr: Domain swapping in inducible nitricoxide synthase. Electron transfer occurs between flavin and heme groups located on adjacent subunits in the dimer. Journal of Biological Chemistry, 273(30), 18950-18958 (1998)

55. C. J. Feng, C. Thomas, M. A. Holliday, G. Tollin, J. C. Salerno, D. K. Ghosh and J. H. Enemark: Direct measurement by laser flash photolysis of intramolecular electron transfer in a two-domain construct of murine inducible nitric oxide synthase. Journal of the American Chemical Society, 128(11), 3808-3811 (2006)

56. C. J. Feng, G. Tollin, M. A. Holliday, C. Thomas, J. C. Salerno, J. H. Enemark and D. K. Ghosh: Intraprotein electron transfer in a two-domain construct of neuronal nitric oxide synthase: The output state in nitric oxide formation. Biochemistry, 45(20), 6354-6362 (2006)

57. D. J. Manstein, V. Massey, S. Ghisla and E. F. Pai: Stereochemistry and accessibility of prosthetic groups in flavoproteins. Biochemistry, 27(7), 2300-2305 (1988)

58. A. Presta, A. M. Weber-Main, M. T. Stankovich and D. J. Stuehr: Comparative effects of substrates and pterin cofactor on the heme midpoint potential in inducible and neuronal nitric oxide synthases. Journal of the American Chemical Society, 120(37), 9460-9465 (1998)

59. A. J. Dunford, K. R. Marshall, A. W. Munro and N. S. Scrutton: Thermodynamic and kinetic analysis of the isolated FAD domain of rat neuronal nitric oxide synthase altered in the region of the FAD shielding residue Phe1395. European Journal of Biochemistry, 271(12), 2548-2560 (2004)

60. K. Panda, S. Ghosh and D. J. Stuehr: Calmodulin activates intersubunit electron transfer in the neuronal nitricoxide synthase dimer. Journal of Biological Chemistry, 276(26), 23349-23356 (2001) 
61. M. J. Bennett, M. P. Schlunegger and D. Eisenberg: 3D Domain Swapping - A Mechanism For Oligomer Assembly. Protein Science, 4(12), 2455-2468 (1995)

62. S. Fuziwara, I. Sagami, E. Rozhkova, D. Craig, M. A. Noble, A. W. Munro, S. K. Chapman and T. Shimizu: Catalytically functional flavocytochrome chimeras of P450BM3 and nitric oxide synthase. Journal of Inorganic Biochemistry, 91(4), 515-526 (2002)

63. C. Feng, G. Tollin, J. T. Hazzard, N. J. Nahm, J. G. Guillemette, J. C. Salerno and D. K. Ghosh: Direct measurement by laser flash photolysis of intraprotein electron transfer in a rat neuronal nitric oxide synthase. Journal of the American Chemical Society, 129(17), 5621-5629 (2007)

64. C. Feng, V. Taiakina, D. K. Ghosh, J. G. Guillemette and G. Tollin: Intraprotein electron transfer between the FMN and heme domains in endothelial nitric oxide synthase holoenzyme. Biochimica Et Biophysica Acta-Proteins and Proteomics, 1814(12), 1997-2002 (2011)

65. C. Feng, A. L. Dupont, N. J. Nahm, D. E. Spratt, J. T. Hazzard, J. B. Weinberg, J. G. Guillemette, G. Tollin and D. K. Ghosh: Intraprotein electron transfer in inducible nitric oxide synthase holoenzyme. Journal of Biological Inorganic Chemistry, 14(1), 133-142 (2009)

66. C. Feng, L. J. Roman, J. T. Hazzard, D. K. Ghosh, G. Tollin and B. S. Masters: Deletion of the autoregulatory insert modulates intraprotein electron transfer in rat neuronal nitric oxide synthase. FEBS Letters, 582(18), 2768-2772 (2008)

67. T. Laursen, A. Singha, N. Rantzau, M. Tutkus, J. Borch, P. Hedegard, D. Stamou, B. L. Moller and N. S. Hatzakis: Single molecule activity measurements of cytochrome P450 oxidoreductase reveal the existence of two discrete functional states. ACS Chemical Biology, 9(3), 630-634 (2014)

68. S. Hay, S. Brenner, B. Khara, A. M. Quinn, S. E. Rigby and N. S. Scrutton: Nature of the energy landscape for gated electron transfer in a dynamic redox protein. Journal of the American Chemical Society, 132(28), 9738-9745 (2010)

69. C. Xia, D. Hamdane, A. L. Shen, V. Choi, C. B. Kasper, N. M. Pearl, H. Zhang, S. Im, L. Waskell and J. P. Kim: Conformational changes of NADPH-cytochrome P450 oxidoreductase are essential for catalysis and cofactor binding. Journal of Biological Chemistry, 286(18), 16246-16260 (2011)

70. D. Hamdane, C. Xia, S. Im, H. Zhang, J. Kim and L. Waskell: Structure and function of an NADPH-cytochrome P450 oxidoreductase in an open conformation capable of reducing cytochrome P450. Journal of Biological Chemistry, 284(17), 11374-11384 (2009)

71. L. Aigrain, D. Pompon, S. Morera and G. Truan: Structure of the open conformation of a functional chimeric NADPH cytochrome P450 reductase. EMBO Reports, 10(7), 242-247 (2009)

72. M. Sugishima, H. Sato, Y. Higashimoto, J. Harada, K. Wada, K. Fukuyama and M. Noguchi: Structural basis for the electron transfer from an open form of NADPH-cytochrome P450 oxidoreductase to heme oxygenase. Proceedings of the National Academy of Sciences of the United States of America, 111(7), 2524-2529 (2014)

73. Y. Dai, M. M. Haque and D. J. Stuehr: Restricting the conformational freedom of the neuronal nitric-oxide synthase flavoprotein domain reveals impact on electron transfer and catalysis. Journal of Biological Chemistry, 292(16), 6753-6764 (2017)

74. V. V. Mozhaev, K. Heremans, J. Frank, P. Masson and C. Balny: High pressure effects on protein structure and function. Proteins-Structure Function and Genetics, 24(1), 81-91 (1996)

75. J. Jonas, L. Ballard and D. Nash: High-resolution, high-pressure NMR studies of proteins. Biophysical Journal, 75(1), 445-452 (1998)

76. J. R. Grigera and A. N. McCarthy: The Behavior of the Hydrophobic Effect under Pressure and Protein Denaturation. Biophysical Journal, 98(8), 1626-1631 (2010)

77. B. R. Crane, A. S. Arvai, D. K. Ghosh, C. Wu, E. D. Getzoff, D. J. Stuehr and J. A. Tainer: Structure of nitric oxide synthase oxygenase dimer with pterin and substrate. Science, 279(5359), 2121-2126 (1998)

78. H. Li, H. Shimizu, M. Flinspach, J. Jamal, W. Yang, M. Xian, T. Cai, E. Z. Wen, Q. Jia, P. G. Wang and T. L. Poulos: The novel binding mode of N-alkyl-N'-hydroxyguanidine to neuronal nitric oxide synthase provides mechanistic insights into NO biosynthesis. Biochemistry, 41(47), 13868-13875 (2002)

79. C. S. Raman, H. Li, P. Martasek, V. Kral, B. S. Masters and T. L. Poulos: Crystal structure of constitutive endothelial nitric oxide synthase: A paradigm for pterin function involving a novel metal center. Cell, 95(7), 939-950 (1998) 80. D. J. Stuehr, J. Santolini, Z. Q. Wang, C. C. Wei and S. Adak: Update on mechanism and catalytic regulation in the NO synthases. Journal of Biological Chemistry, 279(35), 36167-36170 (2004)

81. A. P. Ledbetter, K. McMillan, L. J. Roman, B. S. S. Masters, J. H. Dawson and M. Sono: Low-temperature stabilization and spectroscopic characterization of the dioxygen complex of the ferrous neuronal nitric oxide synthase oxygenase domain. Biochemistry, 38(25), 8014-8021 (1999)

82. C. C. Wei, Z. Q. Wang, A. S. Arvai, C. Hemann, R. Hille, E. D. Getzoff and D. J. Stuehr: Structure of tetrahydrobiopterin tunes its electron transfer to the heme-dioxy intermediate in nitric oxide synthase. Biochemistry, 42(7), 1969-1977 (2003)

83. R. Davydov, A. Ledbetter-Rogers, P. Martasek, M. Larukhin, M. Sono, J. H. Dawson, B. S. S. Masters and B. M. Hoffman: EPR and ENDOR characterization of intermediates in the cryoreduced oxy-nitric oxide synthase heme domain with bound L-arginine or N-G-hydroxyarginine. Biochemistry, 41(33), 10375-10381 (2002)

84. C. C. Wei, Z. Q. Wang, C. Hemann, R. Hille and D. J. Stuehr: A tetrahydrobiopterin radical forms and then becomes reduced during N-omega-hydroxyarginine oxidation by nitric-oxide synthase. Journal of Biological Chemistry, 278(47), 46668-46673 (2003)

85. M. Negrerie, V. Berka, M. H. Vos, U. Liebl, J. C. Lambry, A. L. Tsai and J. L. Martin: Geminate recombination of nitric oxide to endothelial nitric-oxide synthase and mechanistic implications. Journal of Biological Chemistry, 274(35), 24694-24702 (1999) 
86. J. S. Tejero, A. Biswas, Z. Q. Wang, R. C. Page, M. M. Haque, C. Hemann, J. L. Zweier, S. Misra and D. J. Stuehr: Stabilization and Characterization of a Heme-Oxy Reaction Intermediate in Inducible Nitric-oxide Synthase. Journal of Biological Chemistry, 283(48), 33498-33507 (2008)

87. S. Marchal, H. M. Girvan, A. C. F. Gorren, B. Mayer, A. W. Munro, C. Balny and R. Lange: Formation of transient oxygen complexes of cytochrome P450BM3 and nitric oxide synthase under high pressure. Biophysical Journal, 85(5), 3303-3309 (2003)

88. A. C. F. Gorren, N. Bec, A. Schrammel, E. R. Werner, R. Lange and B. Mayer: Low-temperature optical absorption spectra suggest a redox role for tetrahydrobiopterin in both steps of nitric oxide synthase catalysis. Biochemistry, 39(38), 11763-11770 (2000)

89. R. Davydov and B. M. Hoffman: Active intermediates in heme monooxygenase reactions as revealed by cryoreduction/annealing, EPR/ENDOR studies. Archives of Biochemistry and Biophysics, 507(1), 36-43 (2011)

Abbreviations: NOS: nitric oxide synthase; NO: nitric oxide; NADP $(\mathrm{H})$ : nicotinamide adenine dinucleotide phosphate; CaM: calmodulin; FAD: flavin adenine dinucleotide; FMN: flavin mononucleotide; 5-dFMN: 5-deazaflavin mononucleotide; $\mathrm{H}_{4} \mathrm{~B}$ : tetrahydrobiopterin; NOHA: $\mathrm{N}^{\omega}$-hydroxy-L-arginine; cyt $c$ : cytochrome $c$; P450: cytochrome P450; EPR: electron paramagnetic resonance; cryo-EM: cryo-electron microscopy; FRET: Forster resonance energy transfer

Key words: nitric oxide synthase, trapping, protein domain dynamics, thermodynamic block, electron transfer chemistry

Send correspondence to: Nigel Shaun Scrutton, Manchester Institute of Biotechnology, The University of Manchester, Manchester, M1 7DN, United Kingdom, tel, +44 161306 5152, e-mail, nigel.scrutton@ manchester.ac.uk.

\section{FIGURES.}



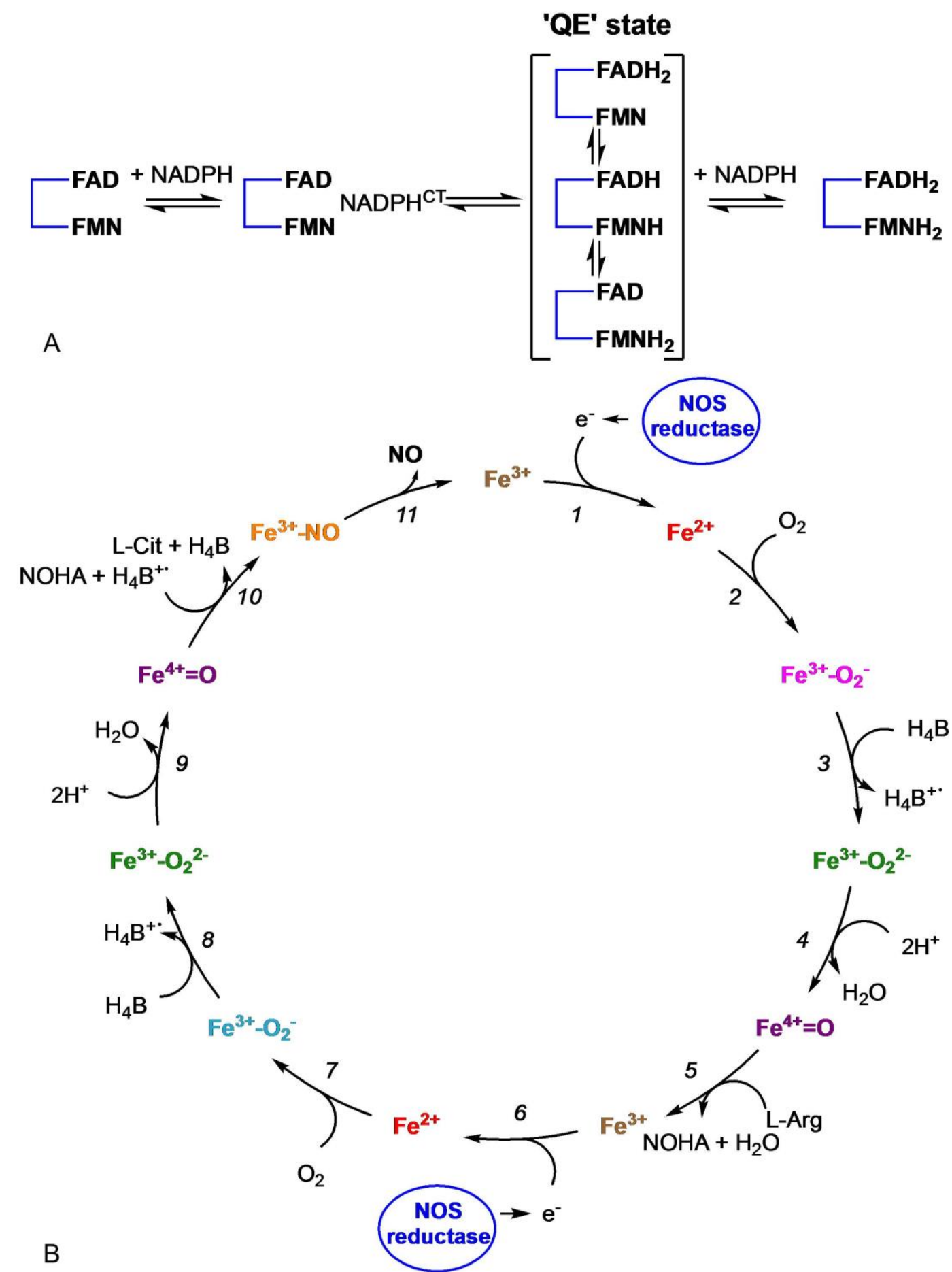

Figure 1. Proposed mechanism of A) NOS catalysed flavin reduction and B) NO generation at the NOS haem oxygenase domain. In $\mathrm{A}$ ), the term ' $\mathrm{QE}$ ' state refers to the quasi-equilibrium state, a form of the enzyme where two electrons are distributed between the FAD and FMN cofactors. 


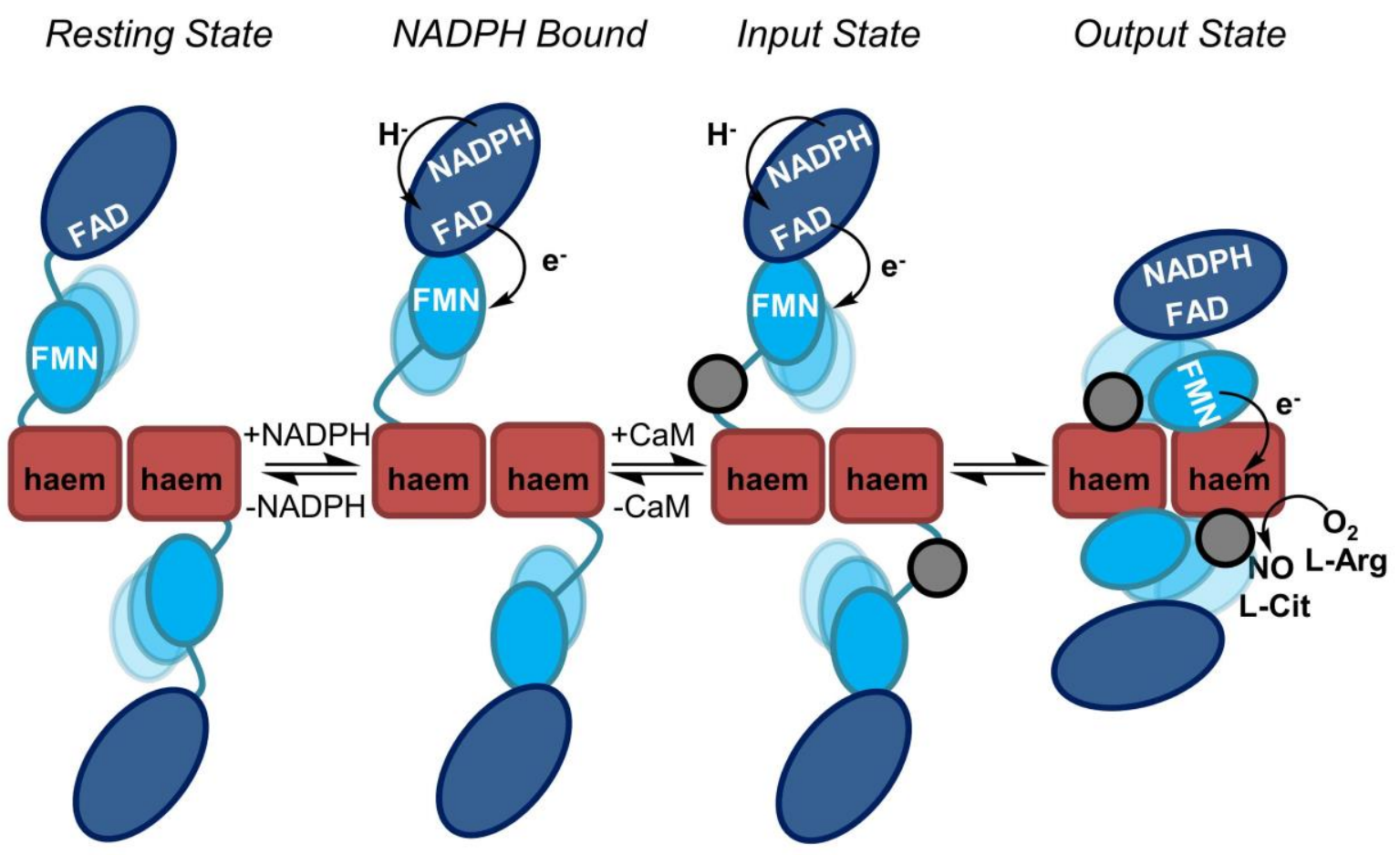

Figure 2. The NOS conformational equilibrium. FAD and connecting domains are shown as dark blue, the FMN domain is shown as light blue, the haem domain is shown as red and $\mathrm{CaM}$ is shown as a grey circle. Electrons, which originate from the NADPH, pass through the NOS FAD and FMN cofactors to the NOS haem oxygenase where dioxygen and L-arginine are converted into NO and L-citrulline.<smiles>Cc1cc2c(cc1C)C(C[C@H](O)[C@H](O)COP(=O)(O)O)C1=NC(=O)NC(=O)C1=N2</smiles>

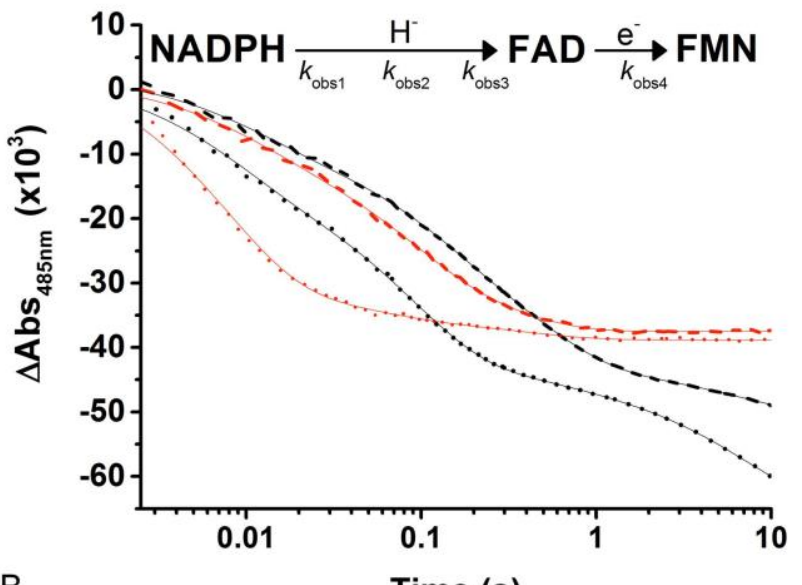

A

B

Time (s)
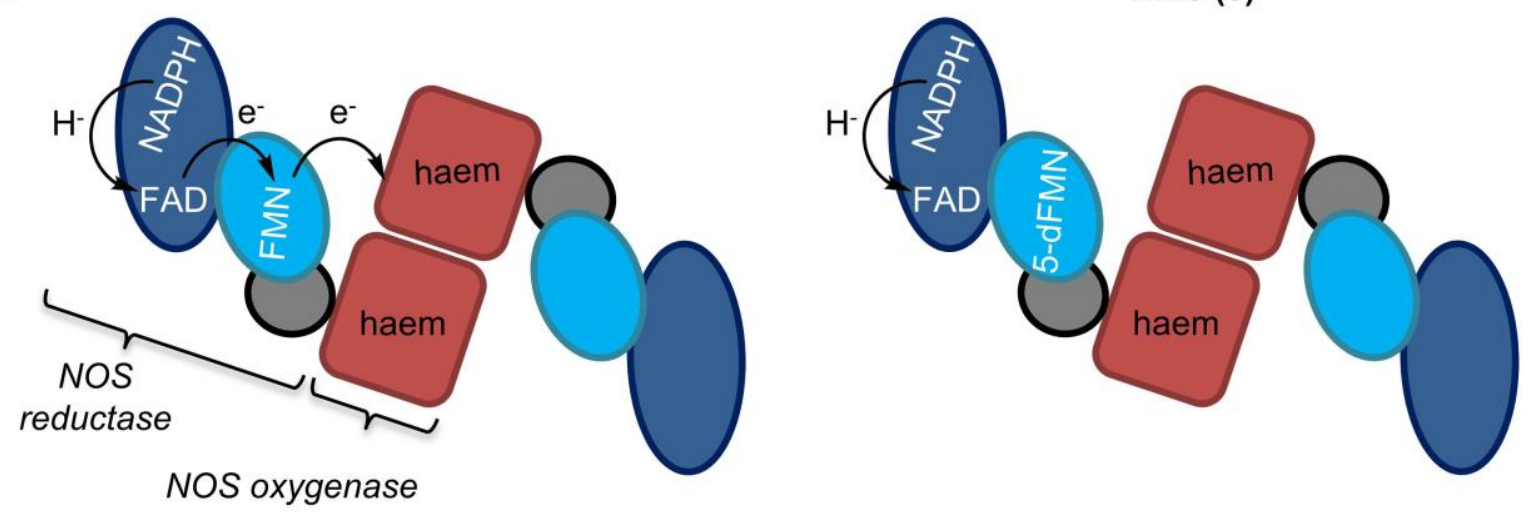

C

nNOS 
Figure 3. Probing inter-flavin electron transfer in NOS using 5-deazaflavin mononucleotide (5-dFMN). A) shows the skeletal structures of FMN (black) and 5-dFMN (red). B) shows example stopped-flow transients for the reactions between NADPH and either full-length wild-type NOS (black) or the 5-dFMN NOS (red) in the presence (dots) and absence (dash) of $\mathrm{CaM}$. The schematic in C) shows the path of electrons from NADPH, through FAD and FMN cofactors, to the NOS haem in wild-type NOS, as well as showing how electron transfer is prevented from FAD to the 5-dFMN in 5-dFMN NOS. In C), the NOS FAD and connecting domains are shown in dark blue, the FMN domain is shown in light blue, the haem domain is shown in red and CaM is shown as a grey circle.

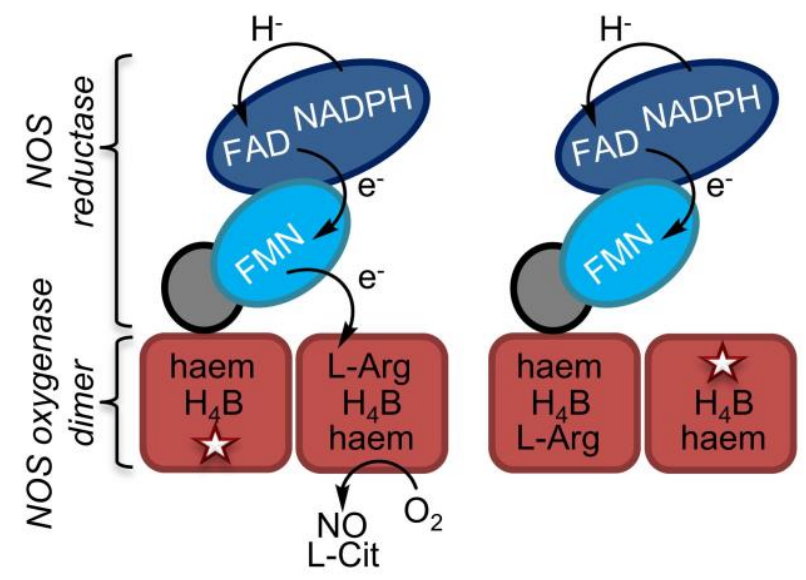

Figure 4. Schematic of the two NOS heterodimers used to probe domain swapping in the NOS family of enzymes. The NOS FAD and connecting domains are shown in dark blue, the FMN domain is shown in light blue, the haem domain is shown in red and CaM is shown as a grey circle. The L-arginine binding knock-out variant is represented in the schematic as a white star.

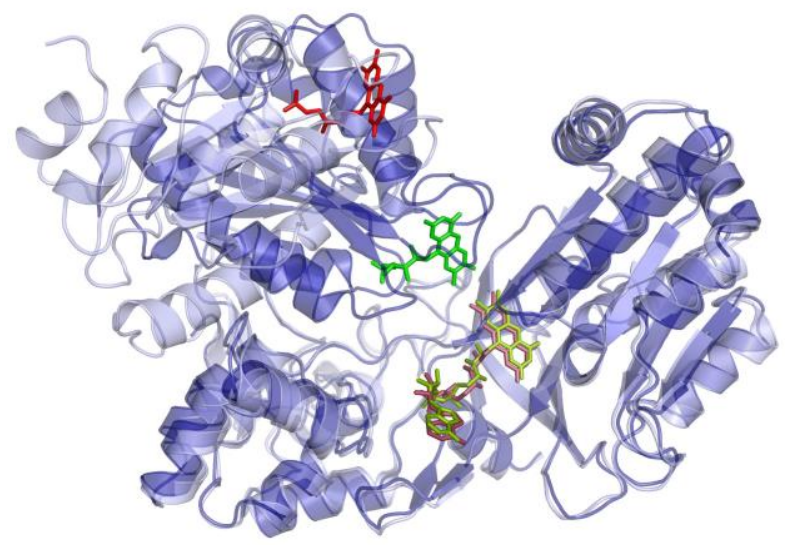

Figure 5. Overlaid structures of 'open' and 'closed' rat CPR. The 'closed' form of CPR (PDB: 1AMO_A) is shown in dark blue cartoon, while the 'open' structure of CPR ( $\triangle$ TGEE CPR;PDB: 3ES9_A) is shown in light blue cartoon. In the 'closed' form of CPR, the FAD and FMN cofactors are shown as light green and green sticks, respectively. Conversely, in the 'open' structure of CPR, the FAD and FMN cofactors are shown as light red and red sticks, respectively. 